\title{
The Role of PGC-1 Coactivators in Aging Skeletal Muscle and Heart
}

\author{
Lloye M. Dillon ${ }^{1}$, Adriana P. Rebelo², and Carlos T. Moraes ${ }^{1,2}$ \\ ${ }^{1}$ Department of Cell Biology and Anatomy, University of Miami Miller School of Medicine, Miami, \\ FL, USA \\ ${ }^{2}$ Department of Neurology, University of Miami Miller School of Medicine, Miami, FL, USA
}

\section{Summary}

Aging is the progressive decline in cellular, tissue, and organ function. This complex process often manifests as loss of muscular strength, cardiovascular function, and cognitive ability.

Mitochondrial dysfunction and decreased mitochondrial biogenesis are believed to participate in metabolic abnormalities and loss of organ function, which will eventually contribute to aging and decreased lifespan. In this review, we discuss what is currently known about mitochondrial dysfunction in the aging skeletal muscle and heart. We focused our discussion on the role of PGC-1 coactivators in the regulation of mitochondrial biogenesis and function and possible therapeutic benefits of increased mitochondrial biogenesis in compensating for mitochondrial dysfunction and circumventing aging and aging-related diseases.

\section{Keywords}

aging; mitochondria; mitochondrial biogenesis; PGC-1; skeletal muscle; heart

\section{INTRODUCTION}

Mitochondria are important for many cellular processes. However, they are primarily responsible for providing cells with energy in the form of adenosine triphosphate (ATP). ATP is produced by oxidative phosphorylation (OXPHOS) using the energy released from electron transfer on the mitochondrial inner membrane. To meet the cell's changing energy requirements, the process of mitochondrial biogenesis needs to regulate mitochondrial protein expression and function in the cell (1). The mitochondrial theory of aging has long proposed mitochondrial dysfunction as a player in the aging process. Recent studies have supported a link between mitochondrial dysfunction associated with aging and decreased mitochondrial biogenesis. A study comparing the transcriptional profile of aging humans to aging mice and flies found that the expression levels of mitochondrial electron transport chain (ETC) components decrease with age in all three organisms (2). This suggests that across species, mitochondrial biogenesis is decreased during aging. 
Over the last few years, the transcriptional coactivators of the peroxisome proliferatoractivated receptor gamma coactivator-1 (PGC-1) family have been shown to be crucial regulators of mitochondrial biogenesis and function $(1,3-6)$. This family includes PGC-1a and PGC-1 $\beta$, which are present in many cell types but more highly expressed in energy demanding tissues such as heart, brain, skeletal muscle and kidney, and PGC-related coactivator (PRC), which is expressed in all tissues $(1,7,8)$. Finally, several independent studies have supported the hypothesis that the PGC-1 protein family is associated with aging. The levels of PGC-1a protein were found to be lower in aged mice than in young mice (9) and loss of PGC-1a has been suggested to be an important contributor to mitochondrial dysfunction in aging-associated diseases (10). Besides, a recent study by Sahin et al. (11) showed that mitochondrial dysfunction in a mouse model of telomere dysfunction, a phenomenon proposed to be a cause of aging, was associated with repressed levels of PGC-1a and PGC-1 $\beta$. This observation revealed a connection between aging in the nucleus and the mitochondria (12) and supported the idea that decreased mitochondrial biogenesis and function are key contributors to aging.

\section{PGC-1 COACTIVATORS AND THE REGULATION OF MITOCHONDRIAL BIOGENESIS}

Mitochondria are composed of $\sim 1,500$ proteins, which are encoded by both the nuclear and mitochondrial DNA (mtDNA). The mtDNA encodes only 13 of these polypeptides, which are subunits of the mitochondrial ETC. It also encodes for two rRNA genes and 22 tRNA genes required for mitochondrial protein synthesis. All other mitochondrial proteins are encoded by the nuclear DNA. Consequently, the process of mitochondrial biogenesis requires coordination between these two genomes. This coordination is regulated primarily by the PGC-1 family of transcriptional coactivators, which work by activating transcription factors. However, they do not interact directly with DNA. As described below, they bind to several different transcription factors that bind DNA. PGC-1 coactivators also interact with a histone acetyltransferase complex that consists of cAMP response element binding protein/ p300 that facilitates chromatin remodeling and also with the thyroid hormone receptorassociated protein/vitamin D receptor-interacting protein (TRAP/DRIP) mediator complex in order to activate DNA transcription (reviewed in refs. 1, 4). In addition to regulating mitochondrial biogenesis, the PGC-1 coactivators are involved in modulating other metabolic pathways including fatty acid oxidation (FAO), lipogenesis, gluconeogenesis, and thermogenesis (reviewed in refs. 1, 4).

The PGC-1 family initiates mitochondrial biogenesis by activating transcription factors that regulate the expression of nuclear DNA encoded mitochondrial proteins (13). These transcription factors include the nuclear respiratory factor 1 and 2 (NRF-1 and NRF-2) and the estrogen related receptor alpha (ERRa) (14-16). The activation of these transcription factors increases the expression of many mitochondrial proteins, including the mitochondrial transcription factor A (TFAM) and the mitochondrial transcription factors b1 and b2 (TFB1M and TFB2M, respectively) $(17,18)$. TFAM is essential for mtDNA replication, transcription, and maintenance $(17,18)$. Therefore, by regulating TFAM levels, the PGC-1 coactivators are able to influence the expression of proteins encoded by the mtDNA. The 
proteins encoded by the nuclear DNA are imported into the mitochondria where some will combine with proteins encoded by the mtDNA to form the multisubunit complexes of the mitochondrial OXPHOS system. Therefore, the PGC-1 family regulates mitochondrial biogenesis by coordinating the expression of mitochondrial proteins encoded by both the nuclear and mitochondrial genomes (Fig. 1).

Although PGC-1a acts mainly in the nucleus, more recently, Aquilano et al. (19) suggested that PGC-1a and the NAD ${ }^{+}$-dependent protein deacetylase sirtuin 1 (SIRT1), another pivotal regulator of mitochondrial biogenesis, are located in the mitochondria. This is an interesting model as before this study, these proteins were thought to function only in the nucleus. Using fluorescent microscopy, the authors showed that PGC-1a and SIRT1 did not only colocalize with the nuclear Hoechst stain but also with cytochrome c, a mitochondrial protein used to label the mitochondrial network (19). In addition, they showed that TFAM interacts with PGC-1a and SIRT1 in the mitochondria to form multiprotein complexes (19). Moreover, they found that the mtDNA D-loop region was present in crosslinked mitochondria immunoprecipitated with anti-PGC-1a and that PGC-1a and SIRT1 were present in nucleoid (a poorly defined structure containing mtDNA and proteins) enriched mitochondrial fractions (19). It will be interesting to confirm if PGC-1a has a mitochondrial localization, whether it is directly involved in the regulation of mtDNA transcription/ replication and whether PGC-1 $\beta$ and PRC are also present in mitochondria.

Despite sharing similar core functions in the regulation of mitochondrial biogenesis, PGC-1 $\beta$ differs from PGC-1a in its mode of regulation. For example, in contrast to PGC-1a, the expression of PGC-1 $\beta$ in brown adipose tissue and skeletal muscle is not increased by cold exposure and exercise $(3,20,21)$. This observation suggests that PGC-1 $\beta$ controls basal mitochondrial biogenesis, while PGC-1a controls mitochondrial activity in response to external stimuli (20). In addition, PGC-1 $1 \alpha$ and PGC-1 $\beta$ are able to coactivate different transcription factors (22). Furthermore, PGC-1 $\alpha$ and PGC-1 $\beta$ have some nonredundant roles, and both are important to maintain optimal body function because their loss seems to be a key factor in mitochondrial dysfunction, aging, and many disease states $(10,20,21,23)$.

Studies performed in mammalian cells and mouse models have highlighted the importance of the PGC-1 family in the regulation of mitochondrial biogenesis. The overexpression of PGC-1a and PGC-1 $\beta$ in C2C12 muscle cells, cardiac myocytes, and primary rat neurons was shown to increase mitochondrial density, the expression of nuclear and mitochondrial genes and mitochondrial function (24-26). Also, increased PGC-1a expression in mouse heart and skeletal muscle triggers an increase in mitochondrial biogenesis $(27,28)$. Accordingly, the ablation of PGC-1a in mouse skeletal muscle and heart leads to decreased expression of most nuclear and mitochondria encoded genes (20), decreased mitochondrial enzyme activity and mitochondria content (29). Similarly, PGC- $1 \beta$ knockout (KO) mice have reduced expression of nuclear and mitochondrial encoded genes important for mitochondrial function and decreased mitochondrial volume in the heart and muscle $(21,30)$. These studies demonstrate that PGC-1 $\alpha$ and PGC- $1 \beta$ play pivotal roles in regulating mitochondrial biogenesis and function. 


\section{MITOCHONDRIAL BIOGENESIS IN THE AGING SKELETAL MUSCLE AND HEART}

In this section, we discuss mitochondrial dysfunction and its implication on aging in the heart and skeletal muscle with emphasis on sarcopenia and cardiovascular diseases. We also discuss the potential benefit of these coactivators in ameliorating aging-associated metabolic dysfunction.

\section{SKELETAL MUSCLE}

\section{Mitochondrial Dysfunction in the Aging Skeletal Muscle}

The skeletal muscle is essential for movement, body posture, and support of internal organs of the body. It is composed of different fiber types (I, IIA, IIB, and IIX) that are classified based on their expression of the myosin heavy chain (MHC) isoforms. Type IIB fibers ("fast glycolytic") are composed of few mitochondria and rely primarily on glycolysis for ATP production to perform fast and powerful activity (31). On the contrary, mitochondria are abundant in type I ("slow oxidative") and also in type IIA and IIX ("fast oxidative/ glycolytic") fibers and produce ATP by OXPHOS making these fibers more fatigue resistant and abundant in glycogen stores (31).

As one ages, there is a slow but increasing loss of skeletal muscle mass and function. This condition, known as sarcopenia, is a world-wide public health concern as it affects the entire aging population and leads to dependence on locomotion aids and increases the incidence of falls and bone fractures (32). Because sarcopenia is such a prevalent condition, many studies have been performed to understand the cellular and molecular mechanisms that are involved in its progression. Over the years, several theories have been proposed to explain sarcopenia. These theories suggest that changes in muscle protein synthesis and degradation, muscle cell apoptosis, denervation/renervation, and endocrine changes contribute to the development of sarcopenia (33).

Several studies have also proposed a contribution of mitochondrial dysfunction to skeletal muscle aging. The abundance of mtDNA and mitochondrial mRNA transcripts and proteins was shown to decrease with age in the skeletal muscle of healthy men and women (34). These changes were associated with an increase in DNA oxidation in the skeletal muscle (34), suggesting that decreased mitochondrial biogenesis leads to mitochondrial dysfunction and increased oxidative DNA damage in the aging skeletal muscle. In another study of aging humans, $20 \%$ to $30 \%$ of skeletal muscle fibers in individuals between the ages of 67 and 92 were shown to have a defect in the mitochondrial OXPHOS (35). This defect was characterized by decreased activity of the nuclear and mitochondrial encoded cytochrome $\mathrm{c}$ oxidase (COX) (complex IV) and increased activity of succinate dehydrogenase (complex II), which is entirely nuclear encoded, suggesting that there is a decrease in mitochondrial gene expression (35). The fibers with a defective OXPHOS also had high levels of mtDNA deletion mutations (35). Additionally, the expression of PGC-1 coactivators and genes involved in mitochondrial OXPHOS were found to be decreased in the skeletal muscle of humans with type 2 diabetes mellitus, which is an age-associated metabolic disease $(36,37)$. Nevertheless, because this mitochondrial dysfunction can also be a consequence of the aging 
process, the contribution of mitochondrial dysfunction to skeletal muscle aging in humans is still a topic of debate (38).

Skeletal muscle aging in rodents is also associated with mitochondrial dysfunction similar to that described in aging humans (39-42). Therefore, rodents are a great model system to study how increased mitochondrial dysfunction in the skeletal muscle leads to muscle fiber loss and sarcopenia. In rats, the age dependent increase in mtDNA mutations and OXPHOS defects in individual skeletal muscle fibers contribute to the splitting, atrophy, and eventual breakage of muscle fibers during aging (39). Recently, it was shown that mice with a proofreading deficient mtDNA polymerase gamma (mtDNA mutator mice) that accumulate mtDNA mutations develop sarcopenia $(43,44)$. These mice have significant skeletal muscle loss, decreased muscle fiber diameter, comprehensive mitochondrial dysfunction, and increased apoptosis (43). In addition, another study showed that the levels of PGC-1a and PGC-1 13 mRNA are dramatically reduced after denervation in rat and mouse skeletal muscle (45). This is an interesting observation as denervation has been proposed to contribute to sarcopenia and other systemic muscle wasting diseases. These studies demonstrate the marked contribution of mitochondrial dysfunction to skeletal muscle aging.

\section{The Role of PGC-1 Coactivators in the Skeletal Muscle}

Because mitochondrial dysfunction was suggested to play a role in skeletal muscle aging, the role of regulators of mitochondrial function in skeletal muscle has become a topic of interest. In particular, the involvement of the PGC-1 coactivators in skeletal muscle physiology has been the focus of many recent studies.

PGC-1a was discovered as a nuclear receptor coactivator whose expression is induced in mouse brown fat and skeletal muscle after cold exposure (7). The expression of PGC-1a in myotubes robustly increases mitochondrial biogenesis and favors uncoupled respiration (17). In the mouse skeletal muscle, PGC-1a mRNA is preferentially expressed in slow twitch (type 1) muscle fibers (46). When overexpressed in mouse skeletal muscle, PGC-1a upregulates mitochondrial biogenesis and induces a change in fiber characteristics to that of oxidative type I and IIA fibers (46). In addition, it was recently shown that increased PGC-1a expression in the mouse skeletal muscle not only increases mitochondrial content but also orchestrates adaptations within each mitochondrion that preferentially enhances fat oxidation favoring the use of fatty acid substrates over carbohydrates for mitochondrial respiration (47). Conversely, skeletal muscle-specific PGC-1a KO mice have diminished mitochondrial gene expression, a shift from oxidative to glycolytic fiber, myopathy, and reduced physical activity along with exercise intolerance (48). Similar to PGC-1a, increased expression of PGC-1 $\beta$ in the mouse skeletal muscle induces mitochondrial biogenesis, upregulates expression of OXPHOS genes and stimulates the formation of type IIX muscle fibers (49). Interestingly, a recent study by Zechner et al. (50) showed that PGC-1 $\beta$ KO in the skeletal muscle of total body PGC-1a KO mice or in wild-type mice has no effect on the amount or distribution of type II fibers in the skeletal muscle but leads to an increase in type I positive fibers. However, they showed that both $\mathrm{KO}$ models had a decrease in the expression of genes important for mitochondrial function (50). These findings highlight the importance of PGC-1 coactivators in regulating mitochondrial function in the skeletal 
muscle but challenge their importance in the regulation of fiber-type switching. Another recent study showed that overexpression of PRC in myotubes increases mitochondrial mass, gene expression, enzyme activity, and respiration though not as robustly as PGC-1a (5). PRC was also shown to induce myoblast proliferation and increase the levels of slow MHC protein (5).

Mechanistically, PGC-1a regulates fiber type switching through the coactivation of the myocyte enhancing factor 2 (MEF2) transcription factor, which is a master regulator of skeletal muscle determination (46, 49). Besides, PGC-1a activates its own expression through the coactivation of MEF2, and this effect is increased by calcineurin, a $\mathrm{Ca}^{2+}$ dependent phosphatase (46). PGC-1a also regulates the expression of hypoxia inducible factor 2 alpha (HIF2a), a transcription factor shown to coordinate switch to slow muscle fibers, through its coactivation of ERRa (51).

The PGC-1 coactivators have also been shown to play an important role in regulating the adaptation of skeletal muscle to exercise. Several studies have demonstrated that exercise induces upregulation of PGC-1a expression in the skeletal muscle of humans and mice via multiple pathways (reviewed in ref. 52). These include the phosphorylation and activation of PGC-1a by AMP-activated protein kinase (AMPK) in response to exercise. PGC-1a can also be phosphorylated and activated by p38 $\gamma$ MAPK (52). When phosphorylated, PGC-1a is deacetylated by SIRT1 leading to its sequestration in the nucleus and increased expression of its target genes $(52,53)$. Subsequently, PGC-1a stimulates mitochondrial biogenesis, fiber-type switching, FAO, and other pathways in the skeletal muscle in response to exercise. Recently, it was shown that exercise stimulates the endoplasmic reticulum (ER) unfolded protein response (UPR) in skeletal muscle (54). The authors proposed that the UPR is involved in regulating metabolic adaptations and protecting the muscle against ER stress associated with exercise (54). Interestingly, they also demonstrated that the UPR response is regulated by PGC-1a through coactivation of ATF6a, a transcription factor known to regulate the UPR (54). This finding further emphasizes the role of PGC-1a in coordinating exercise-induced adaptations in the skeletal muscle.

Safdar et al. (55) recently demonstrated that acute exercise boosts PGC-1a expression in both the nucleus and the mitochondria of mouse skeletal muscle, leading to increased TFAM levels and increased mitochondrial protein levels. This finding not only confirms the mitochondrial localization of PGC-1a but also highlights the importance of exercise in promoting the mitochondrial localization of PGC-1a. Furthermore, others have shown that overexpression of PGC-1a in mouse skeletal muscle increases glucose uptake in the muscle and prevents the depletion of muscle glycogen stores following exercise (56). Besides, skeletal muscle specific PGC-1a KO mice have aberrant glucose homeostasis pointing to the importance of PGC-1a in glucose regulation in skeletal muscle (57).

In times of nutrient and/or oxygen deprivation, the regulation of angiogenesis is crucial to skeletal muscle as it increases blood supply and nutrient delivery to the muscle. The PGC-1 coactivators are important regulators of angiogenesis. During this process, the expression of PGC-1a in the skeletal muscle increases, leading to an upregulation in the expression of the vascular endothelial growth factor (VEGF) and other angiogenic factors (58). This 
angiogenic program mediated by PGC-1 occurs independent of HIF- $1 a$ and through the coactivation of ERRa (58). Additionally, in response to exercise, PGC-1a is activated by $\beta$ adrenergic stimulation and coordinates angiogenesis in the mouse skeletal muscle via this ERRa/VEGF pathway (59). Accordingly, ablation of PGC-1a in the mouse skeletal muscle results in decreased induction of angiogenesis in response to exercise and ischemia $(58,59)$. Lately, Rowe et al. (60) showed that increased expression of PGC-1 $1 \beta$ in mouse skeletal muscle can also induce angiogenesis through ERRa, independently of HIF-1a. However, while both PGC-1 $a$ and PGC-1 $\beta$ robustly induce increased expression of VEGF, they differ in the induction of other angiogenesis related genes (60). Nevertheless, the PGC-1 coactivators have emerged as crucial regulators of skeletal muscle angiogenesis and may therefore be critical for the development of strategies aimed at increasing the blood vessel network in the skeletal muscle.

As described above, PGC-1 coactivators, in particular PGC-1a, are master regulators of skeletal muscle metabolism. They promote increased mitochondrial biogenesis and function, fibertype switching to enrich oxidative fibers, glucose regulation, and angiogenesis in response to exercise and other stimuli. However, other factors are also important for the regulation of these processes. Intriguingly, Narkar et al. (61) recently showed that fiber-type switch from slow glycolytic to fast oxidative fibers along with increase in mitochondrial respiration and angiogenesis can occur in mouse skeletal muscle through ERR $\gamma$ via the AMPK pathway without the contribution of PGC-1a or stimulation from exercise. Nevertheless, the role of PGC-1 coactivators in the regulation of these processes is fundamental to skeletal muscle function.

\section{Benefits of PGC-1 Coactivators and Increased Mitochondrial Biogenesis in the Aging Skeletal Muscle}

The stimulation of mitochondrial biogenesis by increased expression of PGC-1a and/or PGC-1 $\beta$ (PGC-1 $\alpha / \beta)$ can compensate for mitochondrial dysfunction in tissues and cells (6265). Our group showed that increased expression of PGC-1a in the skeletal muscle of aging wild-type mice protects from the development of mitochondrial dysfunction and sarcopenia (28). Wild-type mice with increased muscle PGC-1a also had improved systemic health (28). Increased expression of PGC-1a led to increase in mitochondrial mass, respiration rates, and ATP production in COX deficient MLC1F-Cox $10^{-/-}$mice, a mouse model of mitochondrial muscle myopathy (62). This improvement in mitochondrial function in the skeletal muscle of the mice resulted in a delay in the onset of the myopathy and increase in the animals' life span (62). COX deficiency is a form of mitochondrial dysfunction previously associated with sarcopenia and skeletal muscle aging $(41,66)$. Recently, similar results were obtained by Viscomi et al. (67), who showed that increased PGC-1a expression in the skeletal muscle of Surf1 KO mice, which also have a COX deficiency, increased COX expression and activity, and also increased total mitochondrial biogenesis and function in the skeletal muscle.

In addition to improving mitochondrial function in the aging skeletal muscle, increased expression of PGC-1 coactivators can protect skeletal muscle from protein degradation and atrophy, processes proposed to also contribute to skeletal muscle aging and sarcopenia. It 
was shown that in the skeletal muscle of mice that were either starved or denervated to induce atrophy, increased PGC-1a decreased the expression of genes implicated in protein degradation (68). PGC-1a impeded the activity of FoxO3, a transcription factor involved in regulating the expression of these genes (68). Furthermore, mice with increased PGC-1a were less susceptible to reduction in fiber size, a feature of skeletal muscle atrophy (68). More recently, it was shown that increased expression of PGC- $1 a$ and PGC- $1 \beta$ can retard the progression of protein degradation in normal muscle and also in muscle already undergoing atrophy (69). Both coactivators were able to reduce the proteosomal and lysosomal protein degradation pathways in starved myotubes without affecting the rate of protein synthesis (69). This was proposed to occur by curtailment of the transcription activity of FoxO and also NFkB, a transcription factor known to be important for the induction of atrophy (69).

As discussed earlier, exercise promotes increased PGC-1a expression in the skeletal muscle and thereby regulates many subsequent adaptations in skeletal muscle. There is growing evidence that exercise is a physiological method by which the beneficial effects of PGC-1a can be conferred on the aging skeletal muscle. Our laboratory showed that endurance exercise can increase mitochondrial biogenesis, restore most of the mitochondrial OXPHOS function and delay the onset of mitochondrial myopathy in COX10 KO mice (70). Intriguingly, Safdar et al. (71) recently showed that endurance exercise can reverse the aging phenotype of mtDNA mutator mice. The exercised mtDNA mutator mice had increased PGC-1a and TFAM levels, OXPHOS proteins levels, and COX activity in the skeletal muscle compared with sedentary mtDNA mutator mice (71). This improvement in mitochondrial function in the mouse skeletal muscle was associated with increased endurance (longer time to exhaustion during exercise) and a decrease in the apoptotic index in the skeletal muscle of the mice (71).

Exercise has also been shown to improve the skeletal muscle phenotype of aging humans. Older adults that engage in regular resistance exercise training were shown to have a skeletal muscle transcriptional profile similar to that of young adults in comparison to sedentary older adults (72). The endurance exercise also increased COX activity and reduced oxidative stress in the older adults (72). Recently, it was shown that lifelong endurance exercise not only improves OXPHOS enzyme activity and mitochondrial function in the skeletal muscle of the elderly but it also increases capillarization (angiogenesis) (73). Besides, the skeletal muscle of untrained elderly subjects maintained the ability to respond to exercise by increasing AMPK and p38 mediated phosphorylation events, thus indicating that the elderly skeletal muscle can still respond to exercise induced changes (73).

Increased expression of PGC-1a and mitochondrial biogenesis can also be activated in skeletal muscle by pharmacological agents. These include bezafibrate (a PPAR pan-agonist used in the clinic to treat hyperlipidemia), 5-aminoimidazole-4-carboxamide ribonucleoside (AICAR) (an AMPK agonist) and resveratrol (a naturally occurring compound that may directly or indirectly activate Sirt1) $(62,67,74)$. The effect of bezafibrate on mitochondrial function and biogenesis in skeletal muscle differs between mouse models $(62,67)$. We showed that the administration of bezafibrate to COX deficient MLC1FCox $10^{-/-}$mice increased PGC-1a expression and improved mitochondrial function in the skeletal muscle of 
the mice (62). However, the administration of bezafibrate to Surf KO mice, which have a mild COX deficiency, did not lead to increased PGC-1a expression or increased mitochondrial biogenesis (67). In this model, bezafibrate was found to only induce FAO through activation of PPAR $a$ and PPAR $\beta / \delta$ (67). In addition, bezafibrate had toxic effects on COX deficient ACTA-Cox $15^{-/}$mice that developed a more severe myopathy and became ill as a result of bezafibrate treatment (67). Furthermore, it was recently shown that bezafibrate reduced mitochondrial function and protein expression, while having no effect on the expression of PGC-1a or the PPARs in mice expressing a mutant Twinkle-helicase (deletor mice) (75). Deletor mice develop COX negative fibers late in adulthood and are used as a model of late-onset mitochondrial myopathy (75). However, bezafibrate treated deletor mice had reduced percent COX negative fibers and reduced deletion load in the skeletal muscle (75). They also had increased lipid oxidation and hepatomegaly (75). In fact, bezafibrate has a well-known rodent-specific hepatoproliferative effect, which can cause health complications in mice (76). These results indicate that bezafibrate has varied effects on different mouse models of COX deficiency, and further studies are needed to determine the effects of this drug. AICAR was shown to increase mitochondrial biogenesis and function in the skeletal muscle of three different mouse models of COX deficiency (67). Resveratrol has also been shown to have beneficial effects on the skeletal muscle. Recently, it was shown to impede age-associated increase in oxidative stress in the skeletal muscle of aged mice by mediating a reduction in $\mathrm{H}_{2} \mathrm{O}_{2}$ concentration, lipid peroxidation, and protein carbonyls (77). Despite the benefits of resveratrol, it was unable to ameliorate sarcopenia in these aged mice possibly because it did not increase PGC-1a expression or improve mitochondrial function in the skeletal muscle of the aged mice (77). However, in combination with exercise, resveratrol was shown to improve the endurance of senescenceaccelerated prone mice compared with senescence-accelerated resistant mice and also increases oxygen consumption and the expression of mitochondrial proteins (78).

These findings indicate that PGC-1a activation can confer therapeutic benefits by increasing mitochondrial biogenesis and may be able to circumvent mitochondrial dysfunction and sarcopenia in the aging skeletal muscle.

\section{HEART}

\section{Mitochondrial Dysfunction in the Aging Heart}

Cardiovascular diseases, such as stroke, heart failure, and vascular diseases, are the primary cause of death in the elderly, despite the progress being made in preventative and treatment methods (79). These diseases can result from modifications in heart structure and function during aging. They include: impairment of myocardial diastolic relaxation, $\mathrm{Ca}^{2+}$ signaling/ flux, myofilament function, cardiac energy metabolism, and decrease in cardiomyocytes (reviewed in refs. 79, 80). Many of these aging related changes have been associated with mitochondrial dysfunction in the heart, as mitochondria are the primary energy source in the heart in addition to being involved in several processes such as the regulation of cell death and reactive oxygen species (ROS) production $(80,81)$. Functioning mitochondria are crucial to the maintenance of optimal heart function and the ability of the heart to adapt to changes in physical activity. This is especially true for cardiomyocytes because 
mitochondria account for $\sim 20 \%-30 \%$ of their cell volume (80). In fact, mitochondrial dysfunction and reduced mitochondrial biogenesis have been shown to contribute to cardiomyocytes malfunction and the development of cardiomyopathy (80). It has also been shown that in the aging heart, there is a greater dependence on carbohydrate metabolism for energy (82). This shift in energy source results from an age-associated decrease in the expression of fatty acid metabolism genes (82). Furthermore, reduced expression of PGC-1a was shown to be associated with heart failure in humans $(4,83)$. In addition, studies have shown that there is an increase in the frequency of mtDNA mutations with age in the heart of humans and also in animal models (84). These mtDNA mutations are believed to contribute to the mitochondrial dysfunction associated with aging. Moreover, mtDNA mutator mice, which accumulate mtDNA mutations were shown to develop COX deficiency in cardiomyocytes which most likely contributes to their cardiomyopathy (85). Accordingly, mice harboring a mutant human polymerase $\gamma$ in heart have mitochondrial dysfunction associated with cardiomegaly, biventricular dilation, increased left ventricle mass, cardiac rhythm disturbances, and premature death (86). In another study, mtDNA mutator mice were shown to develop age-associated heart dysfunction, cardiomyopathy, which was linked to decrease in PGC-1a expression and decreased mitochondrial biogenesis (87).

The heart has at least two populations of mitochondria, sub-sarcolemmal mitochondria (SSM), found below the plasma membrane, and interfibrillar mitochondria (IFM) which are surrounded by the myofibrils in the heart (81). These mitochondria differ in function; studies have shown that IFM have higher mitochondrial OXPHOS activity than SSM (81). Interestingly, aged rats were shown to have a decrease in mitochondrial OXPHOS in IFM but not in SSM (88). This OXPHOS deficiency was characterized by marked decrease in COX activity and FAO in the heart (88). Others have shown that OXPHOS deficiency in the IFM is specifically associated with decreased activity of complex III and IV because the activity of complexes I and II did not change with age (81). However, a more recent study showed that there is a progressive decrease in the activity of complexes I, II, III, and IV of the mitochondrial ETC with age in the heart of rats (89). Besides decreased complex activity, there was a gradual increase in lipid peroxidation in the heart of these mice, suggesting a possible contribution of oxidative damaged to their OXPHOS defect and vice versa (89). Accordingly, reductions in mitochondrial antioxidant function along with impaired mitochondrial biogenesis are central to the development of metabolic-related myocardial dysfunction (80).

\section{The Role of PGC-1 Coactivators in the Heart}

The heart is a high energy demanding organ that requires a continuous supply of ATP for proper functioning (90). As discussed above, defects in energy metabolism have been associated with heart diseases and aging (90). The PGC-1 coactivators are crucial regulators of heart energy metabolism. They coactivate several transcription factors (NRF-1, NRF-2, ERRa, and PPARs) in the coordination of mitochondrial biogenesis and function and fatty acid import and use in the heart $(4,90)$. Both, PGC-1a and PGC- $1 \beta$ are highly expressed in heart, and recent studies have shed light on their function in the regulation of heart metabolism $(4,8)$. 
Most of our knowledge on the function of PGC-1 coactivators in the heart came from studies performed in mammalian cells and animal models. Lehman et al. (26) showed that the expression of PGC-1a in the heart increases after birth and in response to fasting, conditions that require an increase in mitochondrial biogenesis. Increased PGC-1a expression coincided with increased expression of PPARa (a known interacting partner of PGC-1a) and PPARa target genes encoding for mitochondrial and FAO enzymes (26). Activation of the PGC-1a-PPARa pathway in heart requires triglycerides and Atgl, an enzyme that regulates the turnover of triglycerides (TAG) (91). These findings indicate that the PPARa/ PGC-1a pathway is important in the regulation of heart energy metabolism.

The overexpression of PGC-1a in rat neonatal cardiac myocytes was shown to induce an increase in mitochondrial biogenesis, oxygen consumption, and coupled respiration (26). However, the overexpression of PGC-1a in postnatal mouse heart leads to unbridled mitochondrial biogenesis that resulted in severe dilated cardiomyopathy (26). Further studies demonstrated that increased expression of PGC-1a in the neonatal heart is beneficial and robustly increases mitochondrial biogenesis, while increased expression of PGC-1a in the adult heart leads to a small increase in mitochondrial biogenesis, aberrant mitochondrial structure, and reversible cardiomyopathy (27). Therefore, although increased mitochondrial biogenesis provides benefits to the heart, it needs to be tightly controlled to prevent adverse effects.

Conversely, mice deficient in PGC-1a have decreased expression of FAO, OXPHOS, and ATP synthesis genes in the heart (20). In addition, lack of PGC-1a caused the heart to become energy deficient and have reduced contractile function in response to external stimuli (20). Besides, another PGC-1a deficient mouse model was shown to have a reduction in the maximal capacity for mitochondrial ATP synthesis and FAO and a buildup of triglycerides due to a reduction in their usage (92). These mice were using glucose as a substrate for oxidation/energy production instead of TAG, a metabolic shift believed to contribute to the development of cardiomyopathy $(92,93)$. These findings point to the importance of PGC-1a in the regulation of mitochondrial function in the heart.

Likewise, mice with global PGC-1 $\beta$ deficiency also have decreased expression of nuclear and mitochondrial encoded genes $(21,30)$ in addition to a weakened heart rate response when challenged (30). In contrast to the relatively mild heart phenotype present in PGC-1a KO and PGC-1 $\beta$ KO mice, ablation of both PGC-1 $\alpha$ and PGC-1 $\beta$ (PGC-1 $\alpha \beta-/-$ mice) resulted in mice with reduced heart size, occasional heart stoppage, low resting heart weight, aberrant mitochondrial structure and density, and severely low cardiac output, which all contribute to their death (a few days after birth) (94). Their phenotype resulted from a cessation of mitochondrial biogenesis in the late fetal stage that prevented the expression of genes needed for the adult stage development (94). These results strongly confirm the importance of the PGC-1 coactivators in the regulation of mitochondrial biogenesis and function in the heart.

\section{Benefits of PGC-1 Coactivators and Increased Mitochondrial Biogenesis in the Aging Heart}

Because PGC-1 coactivators are important for the regulation of mitochondrial biogenesis and energy metabolism in cardiac tissue and reduced expression of these coactivators is a 
feature of age-associated cardiovascular diseases, they make attractive targets for the treatment of heart disease. As discussed in the previous section, increased expression of PGC-1 coactivators and increase in mitochondrial biogenesis confer many benefits to the aging skeletal muscle and is able to slow the progression of sarcopenia. However, the application of this approach in the aging heart requires careful titration of the levels of PGC-1 coactivators as increased PGC-1a expression in the adult heart leads to cardiomyopathy (27). In fact, the therapeutic window lies between high and low levels of PGC-1a in the heart (95).

Caloric restriction, considered to be the most effective anti-aging intervention thus far, is associated with the activation of PGC-1a (13). This is interesting because it was shown that mice subjected to caloric restriction from middle-age had less decrease in the expression of fatty acid metabolism genes and less increase in carbohydrate metabolism in the heart during aging when compared with noncaloric restricted age-matched mice (82). Furthermore, caloric restricted mice had decreased expression of most genes associated with apoptosis in the heart (82). This result suggests that the decrease in cardiomyocytes observed in the aging heart could be due to increased apoptosis associated with mitochondrial dysfunction. These results also indicate that caloric restriction impedes age related transcriptional changes in the heart and may be beneficial in preventing age-associated cardiovascular diseases.

Other methods known to increase PGC-1 coactivators and mitochondrial biogenesis have also been used in an attempt to improve heart function. Studies have shown that exercise, which increases PGC-1a and confers many beneficial effects in the skeletal muscle, can have both positive and negative effects on the human heart depending on its intensity and the health of the subject (96). Nevertheless, exercise therapy is recognized as being important in the prevention and treatment of age-associated cardiovascular diseases (96). However, the effects of exercise on mitochondrial biogenesis and function in the heart are still being unraveled $(97,98)$.

Bezafibrate, as discussed earlier, is a PPAR $(\alpha, \beta / \delta$, and $\gamma)$ pan-agonist that has been shown to increase FAO (67). In addition, it was also shown to increase PGC-1a expression (62, $99)$, mitochondrial biogenesis, and function $(62,63,99)$. Studies in humans have shown that bezafibrate reduces the risk of developing coronary heart disease and myocardial infarction and also reduced the incidence of cardiac mortality in patients with the metabolic syndrome (100). These findings indicate that activation of the PPAR/PGC-1a pathway confers benefits to the aging heart. However, further studies are needed to develop other methods to activate this pathway because bezafibrate has been shown to have low potency and possible toxic effects (100).

\section{CONCLUSION}

Mitochondrial dysfunction seems to play a causal role in aging. The PGC-1 coactivators have emerged as crucial regulators of mitochondrial biogenesis and function and decreases in their expression have been linked to age-associated skeletal muscle and heart dysfunction (Fig. 2). Therefore, increased expression of pathways associated with these coactivators could provide effective improvements of mitochondrial dysfunction during aging. 


\section{Acknowledgments}

This work was supported in part by the National Institutes of Health Grants 1R01AG036871, 5R01CA085700, R56NS041777, and 5R01EY010804, and the Muscular Dystrophy Association.

\section{REFERENCES}

1. Handschin C, Spiegelman BM. Peroxisome proliferator-activated receptor gamma coactivator 1 coactivators, energy homeostasis, and metabolism. Endocr. Rev. 2006; 27:728-735. [PubMed: 17018837]

2. Zahn JM, Sonu R, Vogel H, Crane E, Mazan-Mamczarz K, et al. Transcriptional profiling of aging in human muscle reveals a common aging signature. PLoS Genet. 2006; 2:e115. [PubMed: 16789832]

3. Scarpulla RC. Transcriptional paradigms in mammalian mitochondrial biogenesis and function. Physiol. Rev. 2008; 88:611-638. [PubMed: 18391175]

4. Finck BN, Kelly DP. PGC-1 coactivators: inducible regulators of energy metabolism in health and disease. J. Clin. Invest. 2006; 116:615-622. [PubMed: 16511594]

5. Philp A, Belew MY, Evans A, Pham D, Sivia I, et al. The PGC-1 $\{$ alpha $\}$ related co-activator (PRC) promotes mitochondrial and myogenic adaptations in C2C12 myotubes. Am. J. Physiol. Regul. Integr. Comp. Physiol. 2011; 301:864-872.

6. Mirebeau-Prunier D, Le Pennec S, Jacques C, Gueguen N, Poirier J, et al. Estrogen-related receptor alpha and PGC-1-related coactivator constitute a novel complex mediating the biogenesis of functional mitochondria. FEBS J. 2010; 277:713-725. [PubMed: 20067526]

7. Puigserver P, Wu Z, Park CW, Graves R, Wright M, et al. A cold-inducible coactivator of nuclear receptors linked to adaptive thermogenesis. Cell. 1998; 92:829-839. [PubMed: 9529258]

8. Lin J, Puigserver P, Donovan J, Tarr P, Spiegelman BM. Peroxisome proliferator-activated receptor gamma coactivator 1beta (PGC-1beta), a novel PGC-1-related transcription coactivator associated with host cell factor. J. Biol. Chem. 2002; 277:1645-1648. [PubMed: 11733490]

9. Vina J, Gomez-Cabrera MC, Borras C, Froio T, Sanchis-Gomar F, et al. Mitochondrial biogenesis in exercise and in ageing. Adv. Drug Deliv. Rev. 2009; 61:1369-1374. [PubMed: 19716394]

10. Conley KE, Marcinek DJ, Villarin J. Mitochondrial dysfunction and age. Curr. Opin. Clin. Nutr. Metab. Care. 2007; 10:688-692. [PubMed: 18089948]

11. Sahin E, Colla S, Liesa M, Moslehi J, Muller FL, et al. Telomere dysfunction induces metabolic and mitochondrial compromise. Nature. 2011; 470:359-365. [PubMed: 21307849]

12. Kelly DP. Cell biology: ageing theories unified. Nature. 2011; 470:342-343. [PubMed: 21307852]

13. Anderson R, Prolla T. PGC-1alpha in aging and anti-aging interventions. Biochim. Biophys. Acta. 2009; 1790:1059-1066. [PubMed: 19371772]

14. Virbasius CA, Virbasius JV, Scarpulla RC. NRF-1, an activator involved in nuclear-mitochondrial interactions, utilizes a new DNA-binding domain conserved in a family of developmental regulators. Genes Dev. 1993; 7:2431-2445. [PubMed: 8253388]

15. Virbasius JV, Virbasius CA, Scarpulla RC. Identity of GABP with NRF-2, a multisubunit activator of cytochrome oxidase expression, reveals a cellular role for an ETS domain activator of viral promoters. Genes Dev. 1993; 7:380-392. [PubMed: 8383622]

16. Schreiber SN, Knutti D, Brogli K, Uhlmann T, Kralli A. The transcriptional coactivator PGC-1 regulates the expression and activity of the orphan nuclear receptor estrogen-related receptor alpha (ERRalpha). J. Biol. Chem. 2003; 278:9013-9018. [PubMed: 12522104]

17. Wu Z, Puigserver P, Andersson U, Zhang C, Adelmant G, et al. Mechanisms controlling mitochondrial biogenesis and respiration through the thermogenic coactivator PGC-1. Cell. 1999; 98:115-124. [PubMed: 10412986]

18. Rebelo AP, Dillon LM, Moraes CT. Mitochondrial DNA transcription regulation and nucleoid organization. J. Inherit. Metab. Dis. 2011; 34:941-951. [PubMed: 21541724]

19. Aquilano K, Vigilanza P, Baldelli S, Pagliei B, Rotilio G, et al. Peroxisome proliferator-activated receptor gamma co-activator 1alpha (PGC-1alpha) and sirtuin 1 (SIRT1) reside in mitochondria: 
possible direct function in mitochondrial biogenesis. J. Biol. Chem. 285:21590-21599. [PubMed: 20448046]

20. Arany Z, He H, Lin J, Hoyer K, Handschin C, et al. Transcriptional coactivator PGC-1 alpha controls the energy state and contractile function of cardiac muscle. Cell Metab. 2005; 1:259-271. [PubMed: 16054070]

21. Sonoda J, Mehl IR, Chong LW, Nofsinger RR, Evans RM. PGC-1beta controls mitochondrial metabolism to modulate circadian activity, adaptive thermogenesis, and hepatic steatosis. Proc. Natl. Acad. Sci. USA. 2007; 104:5223-5228. [PubMed: 17360356]

22. Lin J, Handschin C, Spiegelman BM. Metabolic control through the PGC-1 family of transcription coactivators. Cell Metab. 2005; 1:361-370. [PubMed: 16054085]

23. Vianna CR, Huntgeburth M, Coppari R, Choi CS, Lin J, et al. Hypomorphic mutation of PGC-1beta causes mitochondrial dysfunction and liver insulin resistance. Cell Metab. 2006; 4:453-464. [PubMed: 17141629]

24. St-Pierre J, Lin J, Krauss S, Tarr PT, Yang R, et al. Bioenergetic analysis of peroxisome proliferator-activated receptor gamma coactivators 1alpha and 1beta (PGC-1alpha and PGC-1beta) in muscle cells. J. Biol. Chem. 2003; 278:26597-26603. [PubMed: 12734177]

25. Wareski P, Vaarmann A, Choubey V, Safiulina D, Liiv J, et al. PGC-1\{alpha\} and PGC-1\{beta\} regulate mitochondrial density in neurons. J. Biol. Chem. 2009; 284:21379-21385. [PubMed: 19542216]

26. Lehman JJ, Barger PM, Kovacs A, Saffitz JE, Medeiros DM, et al. Peroxisome proliferatoractivated receptor gamma coactivator-1 promotes cardiac mitochondrial biogenesis. J. Clin. Invest. 2000; 106:847-856. [PubMed: 11018072]

27. Russell LK, Mansfield CM, Lehman JJ, Kovacs A, Courtois M, et al. Cardiac-specific induction of the transcriptional coactivator peroxisome proliferator-activated receptor gamma coactivator-1alpha promotes mitochondrial biogenesis and reversible cardiomyopathy in a developmental stage-dependent manner. Circ. Res. 2004; 94:525-533. [PubMed: 14726475]

28. Wenz T, Rossi SG, Rotundo RL, Spiegelman BM, Moraes CT. Increased muscle PGC-1alpha expression protects from sarcopenia and metabolic disease during aging. Proc. Natl. Acad. Sci. USA. 2009; 106:20405-20410. [PubMed: 19918075]

29. Adhihetty PJ, Uguccioni G, Leick L, Hidalgo J, Pilegaard H, et al. The role of PGC-1\{alpha $\}$ on mitochondrial function and apoptotic susceptibility in muscle. Am. J. Physiol. Cell Physiol. 2009; 297:C217-C225. [PubMed: 19439529]

30. Lelliott CJ, Medina-Gomez G, Petrovic N, Kis A, Feldmann HM, et al. Ablation of PGC-1beta results in defective mitochondrial activity, thermogenesis, hepatic function, and cardiac performance. PLoS Biol. 2006; 4:e369. [PubMed: 17090215]

31. Arany Z. PGC-1 coactivators and skeletal muscle adaptations in health and disease. Curr. Opin. Genet. Dev. 2008; 18:426-434. [PubMed: 18782618]

32. Ryall JG, Schertzer JD, Lynch GS. Cellular and molecular mechanisms underlying age-related skeletal muscle wasting and weakness. Biogerontology. 2008; 9:213-228. [PubMed: 18299960]

33. Combaret L, Dardevet D, Bechet D, Taillandier D, Mosoni L, et al. Skeletal muscle proteolysis in aging. Curr. Opin. Clin. Nutr. Metab. Care. 2009; 12:37-41. [PubMed: 19057185]

34. Short KR, Bigelow ML, Kahl J, Singh R, Coenen-Schimke J, et al. Decline in skeletal muscle mitochondrial function with aging in humans. Proc. Natl. Acad. Sci. USA. 2005; 102:5618-5623. [PubMed: 15800038]

35. Bua E, Johnson J, Herbst A, Delong B, McKenzie D, et al. Mitochondrial DNA-deletion mutations accumulate intracellularly to detrimental levels in aged human skeletal muscle fibers. Am. J. Hum. Genet. 2006; 79:469-480. [PubMed: 16909385]

36. Mootha VK, Lindgren CM, Eriksson KF, Subramanian A, Sihag S, et al. PGC-1alpha-responsive genes involved in oxidative phosphorylation are coordinately downregulated in human diabetes. Nat. Genet. 2003; 34:267-273. [PubMed: 12808457]

37. Patti ME, Butte AJ, Crunkhorn S, Cusi K, Berria R, et al. Coordinated reduction of genes of oxidative metabolism in humans with insulin resistance and diabetes: potential role of PGC1 and NRF1. Proc. Natl. Acad. Sci. USA. 2003; 100:8466-8471. [PubMed: 12832613] 
38. Rasmussen UF, Krustrup P, Kjaer M, Rasmussen HN. Experimental evidence against the mitochondrial theory of aging. A study of isolated human skeletal muscle mitochondria. Exp. Gerontol. 2003; 38:877-886. [PubMed: 12915209]

39. Herbst A, Pak JW, McKenzie D, Bua E, Bassiouni M, et al. Accumulation of mitochondrial DNA deletion mutations in aged muscle fibers: evidence for a causal role in muscle fiber loss. J. Gerontol. A Biol. Sci. Med. Sci. 2007; 62:235-245. [PubMed: 17389720]

40. Drew B, Phaneuf S, Dirks A, Selman C, Gredilla R, et al. Effects of aging and caloric restriction on mitochondrial energy production in gastrocnemius muscle and heart. Am. J. Physiol. Regul. Integr. Comp. Physiol. 2003; 284:R474-R480. [PubMed: 12388443]

41. Bua EA, McKiernan SH, Wanagat J, McKenzie D, Aiken JM. Mitochondrial abnormalities are more frequent in muscles undergoing sarcopenia. J. Appl. Physiol. 2002; 92:2617-2624. [PubMed: 12015381]

42. Mansouri A, Muller FL, Liu Y, Ng R, Faulkner J, et al. Alterations in mitochondrial function, hydrogen peroxide release and oxidative damage in mouse hind-limb skeletal muscle during aging. Mech. Ageing Dev. 2006; 127:298-306. [PubMed: 16405961]

43. Hiona A, Sanz A, Kujoth GC, Pamplona R, Seo AY, et al. Mitochondrial DNA mutations induce mitochondrial dysfunction, apoptosis and sarcopenia in skeletal muscle of mitochondrial DNA mutator mice. PLoS One. 5:e11468. [PubMed: 20628647]

44. Kujoth GC, Hiona A, Pugh TD, Someya S, Panzer K, et al. Mitochondrial DNA mutations, oxidative stress, and apoptosis in mammalian aging. Science. 2005; 309:481-484. [PubMed: 16020738]

45. Sacheck JM, Hyatt JP, Raffaello A, Jagoe RT, Roy RR, et al. Rapid disuse and denervation atrophy involve transcriptional changes similar to those of muscle wasting during systemic diseases. FASEB J. 2007; 21:140-155. [PubMed: 17116744]

46. Lin J, Wu H, Tarr PT, Zhang CY, Wu Z, et al. Transcriptional co-activator PGC-1 alpha drives the formation of slow-twitch muscle fibres. Nature. 2002; 418:797-801. [PubMed: 12181572]

47. Hoeks J, Arany Z, Phielix E, Moonen-Kornips E, Hesselink MK, et al. Enhanced lipid -but not carbohydrate- supported mitochondrial respiration in skeletal muscle of PGC-1alpha overexpressing mice. J. Cell. Physiol. 2011

48. Handschin C, Chin S, Li P, Liu F, Maratos-Flier E, et al. Skeletal muscle fiber-type switching, exercise intolerance, and myopathy in PGC-1alpha muscle-specific knock-out animals. J. Biol. Chem. 2007; 282:30014-30021. [PubMed: 17702743]

49. Arany Z, Lebrasseur N, Morris C, Smith E, Yang W, et al. The transcriptional coactivator PGC-1beta drives the formation of oxidative type IIX fibers in skeletal muscle. Cell Metab. 2007; 5:35-46. [PubMed: 17189205]

50. Zechner C, Lai L, Zechner JF, Geng T, Yan Z, et al. Total skeletal muscle PGC-1 deficiency uncouples mitochondrial derangements from fiber type determination and insulin sensitivity. Cell Metab. 12:633-642. [PubMed: 21109195]

51. Rasbach KA, Gupta RK, Ruas JL, Wu J, Naseri E, et al. PGC-1alpha regulates a HIF2alphadependent switch in skeletal muscle fiber types. Proc. Natl. Acad. Sci. USA. 2010; 107:2186621871. [PubMed: 21106753]

52. Lira VA, Benton CR, Yan Z, Bonen A. PGC-1alpha regulation by exercise training and its influences on muscle function and insulin sensitivity. Am. J. Physiol. Endocrinol. Metab. 2010; 299:E145-E161. [PubMed: 20371735]

53. Canto C, Gerhart-Hines Z, Feige JN, Lagouge M, Noriega L, et al. AMPK regulates energy expenditure by modulating NAD1 metabolism and SIRT1 activity. Nature. 2009; 458:1056-1060. [PubMed: 19262508]

54. Wu J, Ruas JL, Estall JL, Rasbach KA, Choi JH, et al. The unfolded protein response mediates adaptation to exercise in skeletal muscle through a PGC-1alpha/ATF6alpha complex. Cell Metab. 13:160-169. [PubMed: 21284983]

55. Safdar A, Little JP, Stokl AJ, Hettinga BP, Akhtar M, et al. Exercise increases mitochondrial PGC-1alpha content and promotes nuclear-mitochondrial cross-talk to coordinate mitochondrial biogenesis. J. Biol. Chem. 2011; 286:10605-10617. [PubMed: 21245132] 
56. Wende AR, Schaeffer PJ, Parker GJ, Zechner C, Han DH, et al. A role for the transcriptional coactivator PGC-1alpha in muscle refueling. J. Biol. Chem. 2007; 282:36642-36651. [PubMed: 17932032]

57. Handschin C, Choi CS, Chin S, Kim S, Kawamori D, et al. Abnormal glucose homeostasis in skeletal muscle-specific PGC-1alpha knockout mice reveals skeletal muscle-pancreatic beta cell crosstalk. J. Clin. Invest. 2007; 117:3463-3474. [PubMed: 17932564]

58. Arany Z, Foo SY, Ma Y, Ruas JL, Bommi-Reddy A, et al. HIF-independent regulation of VEGF and angiogenesis by the transcriptional coactivator PGC-1alpha. Nature. 2008; 451:1008-1012. [PubMed: 18288196]

59. Chinsomboon J, Ruas J, Gupta RK, Thom R, Shoag J, et al. The transcriptional coactivator PGC-1alpha mediates exercise-induced angiogenesis in skeletal muscle. Proc. Natl. Acad. Sci. USA. 2009; 106:21401-21406. [PubMed: 19966219]

60. Rowe GC, Jang C, Patten IS, Arany Z. PGC-1beta regulates angiogenesis in skeletal muscle. Am. J. Physiol. Endocrinol. Metab. 2011; 301:E155-E163. [PubMed: 21364124]

61. Narkar VA, Fan W, Downes M, Yu RT, Jonker JW, et al. Exercise and PGC-1alpha-independent synchronization of type I muscle metabolism and vasculature by ERRgamma. Cell Metab. 2011; 13:283-293. [PubMed: 21356518]

62. Wenz T, Diaz F, Spiegelman BM, Moraes CT. Activation of the PPAR/PGC-1alpha pathway prevents a bioenergetic deficit and effectively improves a mitochondrial myopathy phenotype. Cell Metab. 2008; 8:249-256. [PubMed: 18762025]

63. Bastin J, Aubey F, Rotig A, Munnich A, Djouadi F. Activation of peroxisome proliferatoractivated receptor pathway stimulates the mitochondrial respiratory chain and can correct deficiencies in patients' cells lacking its components. J. Clin. Endocrinol. Metab. 2008; 93:14331441. [PubMed: 18211970]

64. Srivastava S, Barrett JN, Moraes CT. PGC-1alpha/beta upregulation is associated with improved oxidative phosphorylation in cells harboring nonsense mtDNA mutations. Hum. Mol. Genet. 2007; 16:993-1005. [PubMed: 17341490]

65. Srivastava S, Diaz F, Iommarini L, Aure K, Lombes A, et al. PGC-1alpha/beta induced expression partially compensates for respiratory chain defects in cells from patients with mitochondrial disorders. Hum. Mol. Genet. 2009; 18:1805-1812. [PubMed: 19297390]

66. Safdar A, Hamadeh MJ, Kaczor JJ, Raha S, Debeer J, et al. Aberrant mitochondrial homeostasis in the skeletal muscle of sedentary older adults. PLoS One. 5:e10778. [PubMed: 20520725]

67. Viscomi C, Bottani E, Civiletto G, Cerutti R, Moggio M, et al. In vivo correction of COX deficiency by activation of the AMPK/PGC-1alpha axis. Cell Metab. 2011; 14:80-90. [PubMed: 21723506]

68. Sandri M, Lin J, Handschin C, Yang W, Arany ZP, et al. PGC-1alpha protects skeletal muscle from atrophy by suppressing FoxO3 action and atrophy-specific gene transcription. Proc. Natl. Acad. Sci. USA. 2006; 103:16260-16265. [PubMed: 17053067]

69. Brault JJ, Jespersen JG, Goldberg AL. Peroxisome proliferator-activated receptor gamma coactivator 1alpha or 1beta overexpression inhibits muscle protein degradation, induction of ubiquitin ligases, and disuse atrophy. J. Biol. Chem. 285:19460-19471. [PubMed: 20404331]

70. Wenz T, Diaz F, Hernandez D, Moraes CT. Endurance exercise is protective for mice with mitochondrial myopathy. J. Appl. Physiol. 2009; 106:1712-1719. [PubMed: 19286571]

71. Safdar A, Bourgeois JM, Ogborn DI, Little JP, Hettinga BP, et al. Endurance exercise rescues progeroid aging and induces systemic mitochondrial rejuvenation in mtDNA mutator mice. Proc. Natl. Acad. Sci. USA. 2011; 108:4135-4140. [PubMed: 21368114]

72. Melov S, Tarnopolsky MA, Beckman K, Felkey K, Hubbard A. Resistance exercise reverses aging in human skeletal muscle. PLoS One. 2007; 2:e465. [PubMed: 17520024]

73. Iversen N, Krustrup P, Rasmussen HN, Rasmussen UF, Saltin B, et al. Mitochondrial biogenesis and angiogenesis in skeletal muscle of the elderly. Exp. Gerontol. 2011; 46:670-678. [PubMed: 21504786]

74. Puigserver P. Tissue-specific regulation of metabolic pathways through the transcriptional coactivator PGC1-alpha. Int. J. Obes. (Lond.). 2005; 29(Suppl 1):S5-S9. [PubMed: 15711583] 
75. Yatsuga S, Suomalainen A. Effect of bezafibrate treatment on late-onset mitochondrial myopathy in mice. Hum. Mol. Genet. 2012; 21:526-535. [PubMed: 22012983]

76. Monk JP, Todd PA. Bezafibrate. A review of its pharmacodynamic and pharmacokinetic properties, and therapeutic use in hyperlipidaemia. Drugs. 1987; 33:539-576. [PubMed: 3301301]

77. Jackson JR, Ryan MJ, Alway SE. Long-term supplementation with resveratrol alleviates oxidative stress but does not attenuate sarcopenia in aged mice. J. Gerontol. A Biol. Sci. Med. Sci. 66:751764. [PubMed: 21454355]

78. Murase T, Haramizu S, Ota N, Hase T. Suppression of the aging-associated decline in physical performance by a combination of resveratrol intake and habitual exercise in senescenceaccelerated mice. Biogerontology. 2009; 10:423-434. [PubMed: 18830683]

79. Volkova M, Garg R, Dick S, Boheler KR. Aging-associated changes in cardiac gene expression. Cardiovasc. Res. 2005; 66:194-204. [PubMed: 15820188]

80. Ren J, Pulakat L, Whaley-Connell A, Sowers JR. Mitochondrial biogenesis in the metabolic syndrome and cardiovascular disease. J. Mol. Med. (Berl.). 88:993-1001. [PubMed: 20725711]

81. Lesnefsky EJ, Moghaddas S, Tandler B, Kerner J, Hoppel CL. Mitochondrial dysfunction in cardiac disease: ischemia-reperfusion, aging, and heart failure. J. Mol. Cell Cardiol. 2001; 33:1065-1089. [PubMed: 11444914]

82. Lee CK, Allison DB, Brand J, Weindruch R, Prolla TA. Transcriptional profiles associated with aging and middle age-onset caloric restriction in mouse hearts. Proc. Natl. Acad. Sci. USA. 2002; 99:14988-14993. [PubMed: 12419851]

83. Sihag S, Cresci S, Li AY, Sucharov CC, Lehman JJ. PGC-1alpha and ERRalpha target gene downregulation is a signature of the failing human heart. J. Mol. Cell Cardiol. 2009; 46:201-212. [PubMed: 19061896]

84. Wallace DC. Mitochondrial defects in cardiomyopathy and neuromuscular disease. Am. Heart J. 2000; 139:S70-S85. [PubMed: 10650320]

85. Trifunovic A, Wredenberg A, Falkenberg M, Spelbrink JN, Rovio AT, et al. Premature ageing in mice expressing defective mitochondrial DNA polymerase. Nature. 2004; 429:417-423. [PubMed: 15164064]

86. Lewis W, Day BJ, Kohler JJ, Hosseini SH, Chan SS, et al. Decreased mtDNA, oxidative stress, cardiomyopathy, and death from transgenic cardiac targeted human mutant polymerase gamma. Lab. Invest. 2007; 87:326-335. [PubMed: 17310215]

87. Dai DF, Chen T, Wanagat J, Laflamme M, Marcinek DJ, et al. Age-dependent cardiomyopathy in mitochondrial mutator mice is attenuated by overexpression of catalase targeted to mitochondria. Aging Cell. 2010; 9:536-544. [PubMed: 20456298]

88. Fannin SW, Lesnefsky EJ, Slabe TJ, Hassan MO, Hoppel CL. Aging selectively decreases oxidative capacity in rat heart interfibrillar mitochondria. Arch. Biochem. Biophys. 1999; 372:399-407. [PubMed: 10600182]

89. Tatarkova Z, Kuka S, Racay P, Lehotsky J, Dobrota D, et al. Effects of aging on activities of mitochondrial electron transport chain complexes and oxidative damage in rat heart. Physiol. Res. 2011; 60:281-289. [PubMed: 21114360]

90. Rowe GC, Jiang A, Arany Z. PGC-1 coactivators in cardiac development and disease. Circ. Res. 2010; 107:825-838. [PubMed: 20884884]

91. Haemmerle G, Moustafa T, Woelkart G, Buttner S, Schmidt A, et al. ATGL-mediated fat catabolism regulates cardiac mitochondrial function via PPAR-alpha and PGC-1. Nat. Med. 17:1076-1085. [PubMed: 21857651]

92. Lehman JJ, Boudina S, Banke NH, Sambandam N, Han X, et al. The transcriptional coactivator PGC-1alpha is essential for maximal and efficient cardiac mitochondrial fatty acid oxidation and lipid homeostasis. Am. J. Physiol. Heart Circ. Physiol. 2008; 295:H185-H196. [PubMed: 18487436]

93. Madrazo JA, Kelly DP. The PPAR trio: regulators of myocardial energy metabolism in health and disease. J. Mol. Cell Cardiol. 2008; 44:968-975. [PubMed: 18462747]

94. Lai L, Leone TC, Zechner C, Schaeffer PJ, Kelly SM, et al. Transcriptional coactivators PGC-1alpha and PGC-lbeta control overlapping programs required for perinatal maturation of the heart. Genes Dev. 2008; 22:1948-1961. [PubMed: 18628400] 
95. Handschin C. The biology of PGC-1alpha and its therapeutic potential. Trends Pharmacol. Sci. 2009; 30:322-329. [PubMed: 19446346]

96. Fagard RH. Exercise therapy in hypertensive cardiovascular disease. Prog. Cardiovasc. Dis. 53:404-411. [PubMed: 21545926]

97. Li L, Muhlfeld C, Niemann B, Pan R, Li R, et al. Mitochondrial biogenesis and PGC-1alpha deacetylation by chronic treadmill exercise: differential response in cardiac and skeletal muscle. Basic Res. Cardiol. 2011; 106:1221-1234. [PubMed: 21874557]

98. Eisele JC, Schaefer IM, Randel Nyengaard J, Post H, Liebetanz D, et al. Effect of voluntary exercise on number and volume of cardiomyocytes and their mitochondria in the mouse left ventricle. Basic Res. Cardiol. 2008; 103:12-21. [PubMed: 18004633]

99. Wang X, Moraes CT. Increases in mitochondrial biogenesis impair carcinogenesis at multiple levels. Mol. Oncol. 2011; 5:399-409. [PubMed: 21855427]

100. Tenenbaum A, Motro M, Fisman EZ. Dual and panperoxisome proliferator-activated receptors (PPAR) co-agonism: the bezafibrate lessons. Cardiovasc. Diabetol. 2005; 4:14. [PubMed: 16168052] 


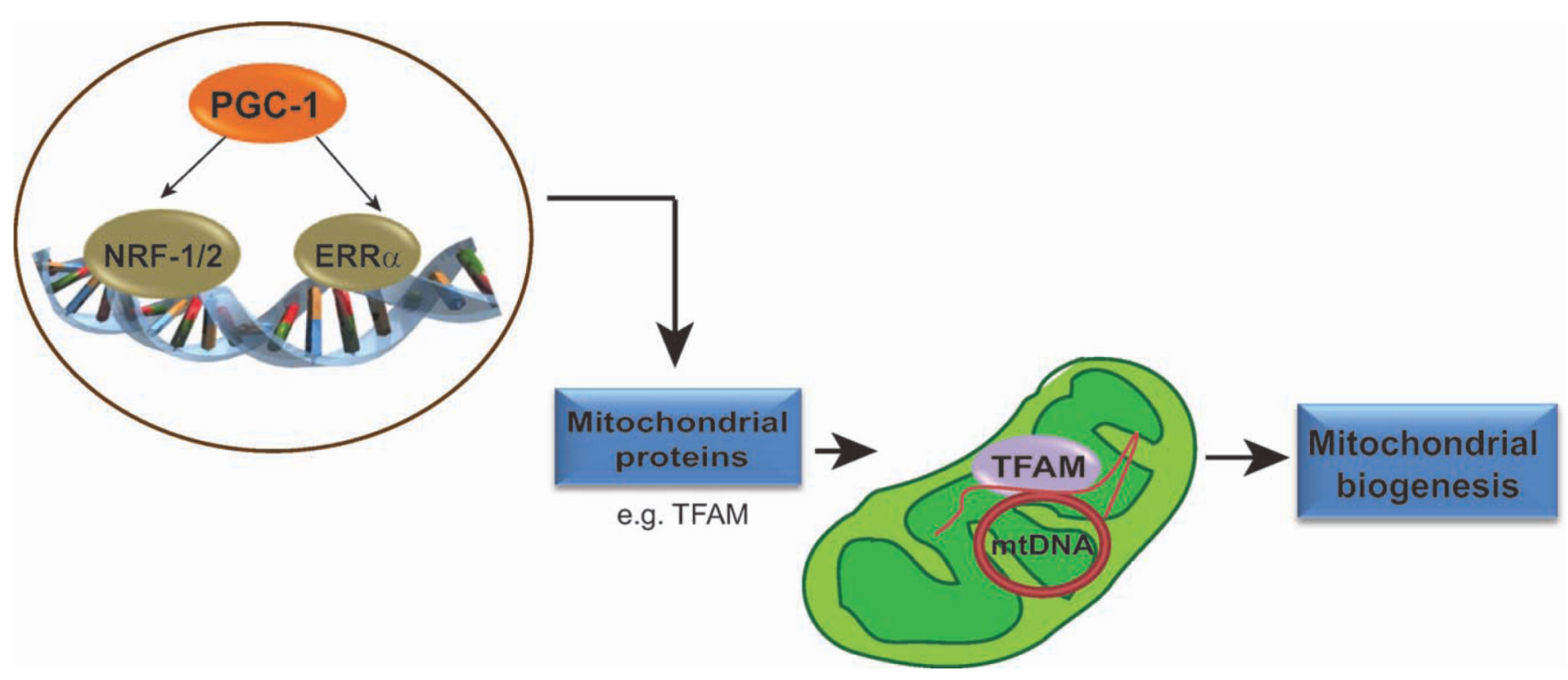

Figure 1.

PGC-1 coactivators are master regulators of mitochondrial biogenesis. PGC-1 coactivators regulate mitochondrial biogenesis by coordinating the expression of mitochondrial proteins encoded by both the nuclear and mitochondrial genome. To accomplish this, they activate transcription factors such as NRF-1/2 and ERRa, thereby increasing the expression of nuclear DNA-encoded mitochondrial proteins. These mitochondrial proteins are then imported into the mitochondria. One such protein is TFAM, which when upregulated, leads to increased mtDNA replication and increased expression of mtDNA-encoded proteins. These processes initiate an increase in mitochondrial biogenesis. 


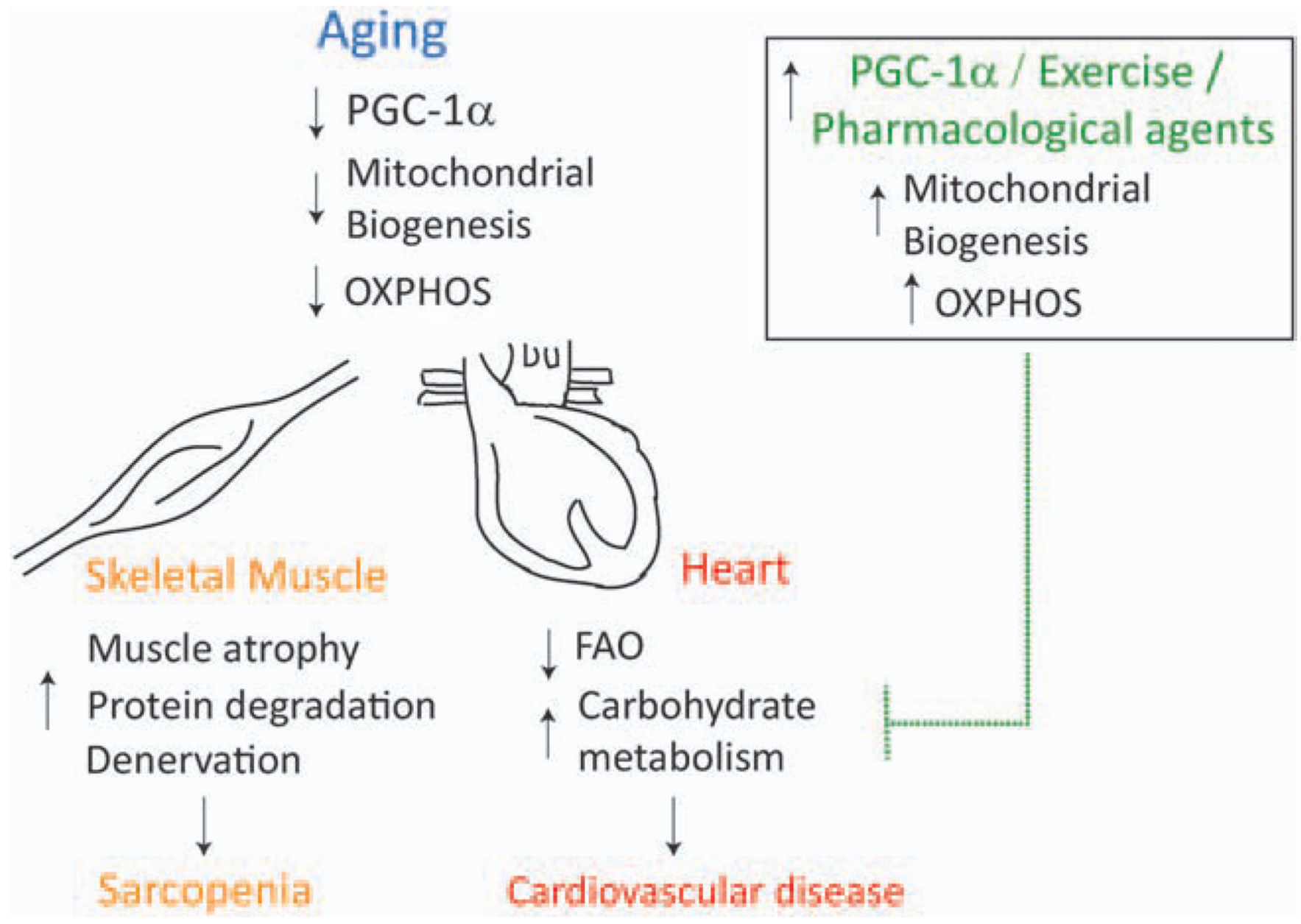

Figure 2.

Decreased PGC-1a expression and mitochondrial dysfunction contribute to skeletal muscle and heart aging. During aging, there is a decrease in PGC-1a expression in the heart and skeletal muscle, which contributes to decrease in mitochondrial protein expression, mtDNA levels, and OXPHOS. This mitochondrial dysfunction is associated with sarcopenia and may contribute to atrophy, protein degradation, and denervation in the skeletal muscle. The adult heart depends primarily on mitochondrial FAO for ATP production. Therefore, decreased PGC-1a expression and mitochondrial dysfunction are believed to contribute to decrease in FAO and increased reliance on carbohydrate metabolism for ATP. This shift in energy metabolism is associated with cardiomyopathy and other cardiovascular diseases. Recent studies have shown that increased PGC-1a expression, whether by exercise/pharmacological agents, leads to increased mitochondrial biogenesis and OXPHOS and may be able to impede the development of sarcopenia and cardiovascular diseases in the aging skeletal muscle and heart, respectively. [Color figure can be viewed in the online issue, which is available at wileyonlinelibrary.com.] 